\title{
TRIBONACCI NUMBERS THAT ARE CONCATENATIONS OF TWO REPDIGITS
}

\author{
MAHADI DDAMULIRA
}

\begin{abstract}
Let $\left(T_{n}\right)_{n>0}$ be the sequence of Tribonacci numbers defined by $T_{0}=0$, $T_{1}=T_{2}=1$, and $T_{n+3}=T_{n+2}+T_{n+1}+T_{n}$ for all $n \geq 0$. In this note, we use of lower bounds for linear forms in logarithms of algebraic numbers and the BakerDavenport reduction procedure to find all Tribonacci numbers that are concatenations of two repdigits.
\end{abstract}

\section{INTRODUCTION}

A repdigit is a positive integer $R$ that has only one distinct digit when written in its decimal expansion. That is, $R$ is of the form

$$
R=\underbrace{\overline{d \cdots d}}_{\ell \text { times }}=d\left(\frac{10^{\ell}-1}{9}\right),
$$

for some positive integers $d, \ell$ with $\ell \geq 1$ and $0 \leq d \leq 9$. The sequence of repdigits is sequence $A 010785$ on the On-Line Encyclopedia of Integer Sequences (OEIS) [6].

Consider the sequence $\left(T_{n}\right)_{n \geq 0}$ of Tribonacci numbers given by

$$
T_{0}=0, \quad T_{1}=1, \quad T_{2}=1, \quad \text { and } \quad T_{n+3}=T_{n+2}+T_{n+1}+T_{n} \quad \text { for all } \quad n \geq 0 .
$$

The sequence of Tribonacci numbers is sequence $A 000073$ on the OEIS. The first few terms of this sequence are given by

$$
\left(T_{n}\right)_{n \geq 0}=0,1,1,2,4,7,13,24,44,81,149,274,504,927,1705,3136,5768,10609,19513, \ldots
$$

\section{Main Result}

In this paper, we study the problem of finding all Tribonacci numbers that are concatenations of two repdigits. More precisely, we completely solve the Diophantine equation

$$
T_{n}=\underbrace{\overline{d_{1} \cdots d_{1}} \underbrace{d_{2} \cdots d_{2}}_{\ell_{2} \text { times }}}_{\ell_{1} \text { times }}=d_{1}\left(\frac{10^{\ell_{1}}-1}{9}\right) \times 10^{\ell_{2}}+d_{2}\left(\frac{10^{\ell_{2}}-1}{9}\right),
$$

in non-negative integers $\left(n, d_{1}, d_{2}, \ell_{1}, \ell_{2}\right)$ with $n \geq 0, \ell_{1} \geq \ell_{2} \geq 1$, and $0 \leq d_{2}<d_{1} \leq 9$.

Our main result is the following.

Theorem 1. The only Tribonacci numbers that are concatenations of two repdigits are

$$
T_{n} \in\{13,24,44,81\} .
$$

2010 Mathematics Subject Classification. Primary 11B39, 11D45; Secondary 11D61, 11J86.

Key words and phrases. Tribonacci number, repdigit, linear form in logarithms, reduction method. 
Our method of proof involves the application of Baker's theory for linear forms in logarithms of algebraic numbers, and the Baker-Davenport reduction procedure. Computations are done with the help of a computer program in Mathematica.

Let $\left(F_{n}\right)_{n>0}$ be the sequence of Fibonacci numbers given by $F_{0}=0, F_{1}=1$, and $F_{n+2}=F_{n+1}+F_{n}$ for all $n \geq 0$, and $\left(B_{n}\right)_{n \geq 0}$ be the sequence of balancing numbers given by $B_{0}=0, B_{1}=1$, and $B_{n+2}=6 B_{n+1}-B_{n}$ for all $n \geq 0$. This paper is inspired by the results of Alahmadi, Altassan, Luca, and Shoaib [1], in which they show that the only Fibonacci numbers that are concatenations of two repdigits are $F_{n} \in\{13,21,34,55,89,144,233,377\}$, and Rayaguru and Panda [7, who showed that $B_{n} \in\{35\}$ is the only balancing number that can be written as a concatenation of two repdigits.

\section{Preliminary Results}

3.1. The Tribonacci sequence. Here, we recall some important properties of the Tribonacci sequence $\left\{T_{n}\right\}_{n \geq 0}$. The characteristic equation

$$
\Psi(x):=x^{3}-x^{2}-x-1=0,
$$

has roots $\alpha, \beta, \gamma=\bar{\beta}$, where

$$
\alpha=\frac{1+\left(r_{1}+r_{2}\right)}{3}, \quad \beta=\frac{2-\left(r_{1}+r_{2}\right)+\sqrt{-3}\left(r_{1}-r_{2}\right)}{6},
$$

and

$$
r_{1}=\sqrt[3]{19+3 \sqrt{33}} \text { and } r_{2}=\sqrt[3]{19-3 \sqrt{33}}
$$

Further, the Binet formula for the general terms of the Tribonacci sequence is given by

$$
T_{n}=a \alpha^{n}+b \beta^{n}+c \gamma^{n} \quad \text { for all } n \geq 0,
$$

where

$$
a=\frac{1}{(\alpha-\beta)(\alpha-\gamma)}, \quad b=\frac{1}{(\beta-\alpha)(\beta-\gamma)}, \quad c=\frac{1}{(\gamma-\alpha)(\gamma-\beta)}=\bar{b}
$$

Furthermore,

$$
a=\frac{\alpha}{\alpha^{2}+2 \alpha+3}
$$

and the minimal polynomial of $a$ over the integers is given by

$$
44 x^{3}+4 x-1
$$

has zeros $a, b, c$ with $\max \{|a|,|b|,|c|\}<1$. Numerically, the following estimates hold:

$$
\begin{gathered}
1.83<\alpha<1.84 \\
0.73<|\beta|=|\gamma|=\alpha^{-\frac{1}{2}}<0.74 \\
0.18<a<0.19 \\
0.35<|b|=|c|<0.36 .
\end{gathered}
$$

From (3), (4), and (7), it is easy to see that the contribution the complex conjugate roots $\beta$ and $\gamma$, to the right-hand side of (5), is very small. In particular, setting

$$
e(n):=T_{n}-a \alpha^{n}=b \beta^{n}+c \gamma^{n} \quad \text { then } \quad|e(n)|<\frac{1}{\alpha^{n / 2}},
$$


holds for all $n \geq 1$. Furthermore, by induction, one can prove that

$$
\alpha^{n-2} \leq T_{n} \leq \alpha^{n-1} \quad \text { holds for all } \quad n \geq 1 .
$$

Let $\mathbb{K}:=\mathbb{Q}(\alpha, \beta)$ be the splitting field of the polynomial $\Psi$ over $\mathbb{Q}$. Then, $[\mathbb{K}, \mathbb{Q}]=6$. Furthermore, $[\mathbb{Q}(\alpha): \mathbb{Q}]=3$. The Galois group of $\mathbb{K}$ over $\mathbb{Q}$ is given by

$$
\mathcal{G}:=\operatorname{Gal}(\mathbb{K} / \mathbb{Q}) \cong\{(1),(\alpha \beta),(\alpha \gamma),(\beta \gamma),(\alpha \beta \gamma),(\alpha \gamma \beta)\} \cong S_{3}
$$

Thus, we identify the automorphisms of $\mathcal{G}$ with the permutations of the zeros of the polynomial $\Psi$. For example, the permutation $(\alpha \gamma)$ corresponds to the automorphism $\sigma: \alpha \rightarrow \gamma, \gamma \rightarrow \alpha, \beta \rightarrow \beta$.

3.2. Linear forms in logarithms. Let $\eta$ be an algebraic number of degree $d$ with minimal primitive polynomial over the integers

$$
a_{0} x^{d}+a_{1} x^{d-1}+\cdots+a_{d}=a_{0} \prod_{i=1}^{d}\left(x-\eta^{(i)}\right),
$$

where the leading coefficient $a_{0}$ is positive and the $\eta^{(i)}$ 's are the conjugates of $\eta$. Then the logarithmic height of $\eta$ is given by

$$
h(\eta):=\frac{1}{d}\left(\log a_{0}+\sum_{i=1}^{d} \log \left(\max \left\{\left|\eta^{(i)}\right|, 1\right\}\right)\right) .
$$

In particular, if $\eta=p / q$ is a rational number with $\operatorname{gcd}(p, q)=1$ and $q>0$, then $h(\eta)=$ $\log \max \{|p|, q\}$. The following are some of the properties of the logarithmic height function $h(\cdot)$, which will be used in the next section of this paper without reference:

$$
\begin{aligned}
h\left(\eta_{1} \pm \eta_{2}\right) & \leq h\left(\eta_{1}\right)+h\left(\eta_{2}\right)+\log 2 ; \\
h\left(\eta_{1} \eta_{2}^{ \pm 1}\right) & \leq h\left(\eta_{1}\right)+h\left(\eta_{2}\right) ; \\
h\left(\eta^{s}\right) & =|s| h(\eta) \quad(s \in \mathbb{Z}) .
\end{aligned}
$$

We recall the result of Bugeaud, Mignotte, and Siksek ([2], Theorem 9.4, pp. 989), which is a modified version of the result of Matveev [5], which is one of our main tools in this paper.

Theorem 2. Let $\eta_{1}, \ldots, \eta_{t}$ be positive real algebraic numbers in a real algebraic number field $\mathbb{K} \subset \mathbb{R}$ of degree $D, b_{1}, \ldots, b_{t}$ be nonzero integers, and assume that

$$
\Lambda:=\eta_{1}^{b_{1}} \cdots \eta_{t}^{b_{t}}-1 \neq 0
$$

Then,

$$
\log |\Lambda|>-1.4 \times 30^{t+3} \times t^{4.5} \times D^{2}(1+\log D)(1+\log B) A_{1} \cdots A_{t},
$$

where

$$
B \geq \max \left\{\left|b_{1}\right|, \ldots,\left|b_{t}\right|\right\}
$$

and

$$
A_{i} \geq \max \left\{D h\left(\eta_{i}\right),\left|\log \eta_{i}\right|, 0.16\right\}, \quad \text { for all } \quad i=1, \ldots, t \text {. }
$$


3.3. Reduction procedure. During the calculations, we get upper bounds on our variables which are too large, thus we need to reduce them. To do so, we use some result from the theory of continued fractions. For a nonhomogeneous linear form in two integer variables, we use a slight variation of a result due to Dujella and Pethő ([3], Lemma 5a). For a real number $X$, we write $\|X\|:=\min \{|X-n|: n \in \mathbb{Z}\}$ for the distance from $X$ to the nearest integer.

Lemma 1. Let $M$ be a positive integer, $\frac{p}{q}$ be a convergent of the continued fraction expansion of the irrational number $\tau$ such that $q>6 M$, and $A, B, \mu$ be some real numbers with $A>0$ and $B>1$. Furthermore, let $\varepsilon:=\|\mu q\|-M\|\tau q\|$. If $\varepsilon>0$, then there is no solution to the inequality

$$
0<|u \tau-v+\mu|<A B^{-w},
$$

in positive integers $u, v$, and $w$ with

$$
u \leq M \quad \text { and } \quad w \geq \frac{\log (A q / \varepsilon)}{\log B} .
$$

The following Lemma is also useful. It is due to Gúzman Sánchez and Luca (4], Lemma 7).

Lemma 2. If $r \geq 1, H>\left(4 r^{2}\right)^{r}$, and $H>L /(\log L)^{r}$, then

$$
L<2^{r} H(\log H)^{r} \text {. }
$$

\section{The Proof of Theorem 1}

4.1. The small ranges. With the help of Mathematica, we checked all the solutions to the Diophantine equation (2) in the ranges $0 \leq d_{2}<d_{1} \leq 9$ and $1 \leq \ell_{2} \leq \ell_{1} \leq n \leq 200$ and found only the solutions stated in Theorem 1. From now on we assume that $n>200$.

4.2. The initial bound on $n$. We rewrite $(2)$ as

$$
\begin{aligned}
T_{n} & =\underbrace{\overline{d_{1} \cdots d_{1}} \underbrace{d_{2} \cdots d_{2}}_{\ell_{2} \text { times }}}_{\ell_{1} \text { times }} \\
& =\underbrace{\overline{d_{1} \cdots d_{1}}}_{\ell_{1} \text { times }} \times 10^{\ell_{2}}+\underbrace{\overline{d_{2} \cdots d_{2}}}_{\ell_{2} \text { times }} \\
& =d_{1}\left(\frac{10^{\ell_{1}}-1}{9}\right) \times 10^{\ell_{2}}+d_{2}\left(\frac{10^{\ell_{2}}-1}{9}\right) \quad(\text { by }(1)) \\
& =\frac{1}{9}\left(d_{1} \times 10^{\ell_{1}+\ell_{2}}-\left(d_{1}-d_{2}\right) \times 10^{\ell_{2}}-d_{2}\right) .
\end{aligned}
$$

Thus,

$$
T_{n}=\frac{1}{9}\left(d_{1} \times 10^{\ell_{1}+\ell_{2}}-\left(d_{1}-d_{2}\right) \times 10^{\ell_{2}}-d_{2}\right) .
$$

We prove the following lemma, which gives a relation on the size of $n$ versus $\ell_{1}+\ell_{2}$.

Lemma 3. All solutions of the Diophantine equation (10) satisfy

$$
\left(\ell_{1}+\ell_{2}\right) \log 10-2<n \log \alpha<\left(\ell_{1}+\ell_{2}\right) \log 10+2 .
$$


Proof. The proof follows easily from (9). One can see that

$$
\alpha^{n-2} \leq T_{n}<10^{\ell_{1}+\ell_{2}} .
$$

Taking the logarithm on both sides, we get that

$$
(n-2) \log \alpha<\left(\ell_{1}+\ell_{2}\right) \log 10,
$$

which leads to

$$
n \log \alpha<\left(\ell_{1}+\ell_{2}\right) \log 10+2 \log \alpha<\left(\ell_{1}+\ell_{2}\right) \log 10+2 .
$$

For the lower bound, we have that

$$
10^{\ell_{1}+\ell_{2}-1}<T_{n} \leq \alpha^{n-1} .
$$

Taking the logarithm on both sides, we get that

$$
\left(\ell_{1}+\ell_{2}-1\right) \log 10<(n-1) \log \alpha,
$$

which leads to

$$
\left(\ell_{1}+\ell_{2}\right) \log 10-2<\left(\ell_{1}+\ell_{2}-1\right) \log 10+\log \alpha<n \log \alpha .
$$

Comparing (11) and (12) gives the result in the lemma.

Next, we examine 10$)$ in two different steps.

Step 1. Substituting (5) in (10), we get that

$$
a \alpha^{n}+b \beta^{n}+c \gamma^{n}=\frac{1}{9}\left(d_{1} \cdot 10^{\ell_{1}+\ell_{2}}-\left(d_{1}-d_{2}\right) \cdot 10^{\ell_{2}}-d_{2}\right) .
$$

By (8), this is equivalent to

$$
9 a \alpha^{n}-d_{1} \cdot 10^{\ell_{1}+\ell_{2}}=-9 e(n)-\left(d_{1}-d_{2}\right) \cdot 10^{\ell_{2}}-d_{2},
$$

from which we deduce that

$$
\begin{aligned}
\left|9 a \alpha^{n}-d_{1} \cdot 10^{\ell_{1}+\ell_{2}}\right| & =\left|9 e(n)+\left(d_{1}-d_{2}\right) \cdot 10^{\ell_{2}}+d_{2}\right| \\
& \leq 9 \alpha^{-n / 2}+9 \cdot 10^{\ell_{2}}+9 \\
& <28 \times 10^{\ell_{2}} .
\end{aligned}
$$

Thus, dividing both sides by $d_{1} \cdot 10^{\ell_{1}+\ell_{2}}$ we get that

$$
\left|\left(\frac{9 a}{d_{1}}\right) \cdot \alpha^{n} \cdot 10^{-\ell_{1}-\ell_{2}}-1\right|<\frac{28 \times 10^{\ell_{2}}}{d_{1} \cdot 10^{\ell_{1}+\ell_{2}}}<\frac{28}{10^{\ell_{1}}} .
$$

Put

$$
\Lambda_{1}:=\left(\frac{9 a}{d_{1}}\right) \cdot \alpha^{n} \cdot 10^{-\ell_{1}-\ell_{2}}-1 .
$$

Next, we apply Theorem 2 on (14). First, we need to check that $\Lambda_{1} \neq 0$. If it were, then we would get that

$$
a \alpha^{n}=\frac{d_{1}}{9} \cdot 10^{\ell_{1}+\ell_{2}}
$$


Now, we apply the automorphism $\sigma$ of the Galois group $\mathcal{G}$ on both sides and take absolute values as follows.

$$
\left|\frac{d_{1}}{9} \cdot 10^{\ell_{1}+\ell_{2}}\right|=\left|\sigma\left(a \alpha^{n}\right)\right|=\left|c \gamma^{n}\right|<1,
$$

which is false. Thus, $\Lambda_{1} \neq 0$. So, we apply Theorem 2 on 14 with the data:

$$
t:=3, \quad \eta_{1}:=\frac{9 a}{d_{1}}, \quad \eta_{2}:=\alpha, \quad \eta_{3}:=10, \quad b_{1}:=1, \quad b_{2}:=n, \quad b_{3}:=-\ell_{1}-\ell_{2} .
$$

By Lemma 3, we have that $\ell_{1}+\ell_{2}<n$. Therefore, we can take $B:=n$. Observe that $\mathbb{K}:=\mathbb{Q}\left(\eta_{1}, \eta_{2}, \eta_{3}\right)=\mathbb{Q}(\alpha)$, since $a=\alpha /\left(\alpha^{2}+2 \alpha+3\right)$, so $D:=3$. We have

$$
h\left(\eta_{1}\right)=h\left(9 a / d_{1}\right) \leq h(9)+h(a)+h\left(d_{1}\right) \leq \log 9+\frac{1}{3} \log 44+\log 9 \leq 5.66 .
$$

Furthermore, $h\left(\eta_{2}\right)=h(\alpha)=(1 / 3) \log \alpha$ and $h\left(\eta_{3}\right)=h(10)=\log 10$. Thus, we can take

$$
A_{1}:=16.98, \quad A_{2}:=\log \alpha, \quad \text { and } \quad A_{3}:=3 \log 10 .
$$

Theorem 2 tells us that

$$
\begin{aligned}
\log \left|\Lambda_{1}\right| & >-1.4 \times 30^{6} \times 3^{4.5} \times 3^{2}(1+\log 3)(1+\log n)(16.98)(\log \alpha)(3 \log 10) \\
& >-1.94 \times 10^{14}(1+\log n) .
\end{aligned}
$$

Comparing the above inequality with $(13)$ gives

$$
\ell_{1} \log 10-\log 28<1.94 \times 10^{14}(1+\log n),
$$

leading to

$$
\ell_{1} \log 10<1.96 \times 10^{14}(1+\log n) .
$$

Step 2. By (8), we rewrite 10 as

$$
9 a \alpha^{n}-\left(d_{1} \cdot 10^{\ell_{1}}-\left(d_{1}-d_{2}\right)\right) \cdot 10^{\ell_{2}}=-9 e(n)-d_{2},
$$

from which we deduce that

$$
\left|9 a \alpha^{n}-\left(d_{1} \cdot 10^{\ell_{1}}-\left(d_{1}-d_{2}\right)\right) \cdot 10^{\ell_{2}}\right|=\left|9 e(n)+d_{2}\right| \leq 9 \alpha^{-n / 2}+9<18 .
$$

Thus, dividing both sides by $9 a \alpha^{n}$ we get that

$$
\left|\left(\frac{d_{1} \cdot 10^{\ell_{1}}-\left(d_{1}-d_{2}\right)}{9 a}\right) \cdot \alpha^{-n} \cdot 10^{\ell_{2}}-1\right|<\frac{18}{9 a \alpha^{n}}<\frac{2}{\alpha^{n}} .
$$

Put

$$
\Lambda_{2}:=\left(\frac{d_{1} \cdot 10^{\ell_{1}}-\left(d_{1}-d_{2}\right)}{9 a}\right) \cdot \alpha^{-n} \cdot 10^{\ell_{2}}-1 .
$$

Next, we apply Theorem 2 on $(17)$. First, we need to chech that $\Lambda_{2} \neq 0$. If not, then we would get that

$$
a \alpha^{n}=\left(\frac{d_{1} \cdot 10^{\ell_{1}}-\left(d_{1}-d_{2}\right)}{9}\right) \cdot 10^{\ell_{2}} .
$$


As before, we apply the automorphism $\sigma$ of the Galois group $\mathcal{G}$ on both sides and take absolute values as follows.

$$
\left|\left(\frac{d_{1} \cdot 10^{\ell_{1}}-\left(d_{1}-d_{2}\right)}{9}\right) \cdot 10^{\ell_{2}}\right|=\left|\sigma\left(a \alpha^{n}\right)\right|=\left|c \gamma^{n}\right|<1
$$

which is false. Thus, $\Lambda_{2} \neq 0$. So, we apply Theorem 2 on 17$)$ with the data:

$t:=3, \quad \eta_{1}:=\frac{d_{1} \cdot 10^{\ell_{1}}-\left(d_{1}-d_{2}\right)}{9 a}, \quad \eta_{2}:=\alpha, \quad \eta_{3}:=10, \quad b_{1}:=1, \quad b_{2}:=-n, \quad b_{3}:=\ell_{2}$.

As before, we have that $\ell_{2}<n$. Thus, we can take $B:=n$. Similary, $\mathbb{Q}\left(\eta_{1}, \eta_{2}, \eta_{3}\right)=\mathbb{Q}(\alpha)$, so we take $D:=3$. Furthermore, we have

$$
\begin{aligned}
h\left(\eta_{1}\right) & =h\left(\frac{d_{1} \cdot 10^{\ell_{1}}-\left(d_{1}-d_{2}\right)}{9 a}\right) \\
& \leq h\left(d_{1} \times 10^{\ell_{1}}-\left(d_{1}-d_{2}\right)\right)+h(9 a) \\
& \leq h\left(d_{1} \times 10^{\ell_{1}}\right)+h\left(d_{1}-d_{2}\right)+h(9)+h(a)+\log 2 \\
& \leq h\left(d_{1}\right)+\ell_{1} h(10)+h\left(d_{1}\right)+h\left(d_{2}\right)+h(9)+h(a)+2 \log 2 \\
& \leq \ell_{1} \log 10+4 \log 9+\frac{1}{3} \log 44+2 \log 2 \\
& \leq 1.96 \times 10^{14}(1+\log n)+4 \log 9+\frac{1}{3} \log 44+2 \log 2 \quad(\text { by } 15) \\
& <1.98 \times 10^{14}(1+\log n) .
\end{aligned}
$$

Thus, we can take

$$
A_{1}:=5.94 \times 10^{14}(1+\log n), \quad A_{2}:=\log \alpha, \quad \text { and } \quad A_{3}:=3 \log 10 .
$$

Theorem 2 tells us that

$$
\begin{aligned}
\log \left|\Lambda_{2}\right| & >-1.4 \times 30^{6} \times 3^{4.5} \times 3^{2}(1+\log 3)(1+\log n)\left(5.94 \times 10^{14}(1+\log n)\right)(\log \alpha)(3 \log 10) \\
& >-6.77 \times 10^{27}(1+\log n)^{2} .
\end{aligned}
$$

Comparing the above inequality with 16 gives,

$$
n \log \alpha-\log 2<6.77 \times 10^{27}(1+\log n)^{2},
$$

which is equivalent to

$$
n<2.24 \times 10^{28}(\log n)^{2} .
$$

Applying Lemma 2 on (18) with the data $r=2, H:=2.24 \times 10^{28}$, and $L:=n$, gives

$$
n<1.22 \times 10^{32} \text {. }
$$

Lemma 3 implies that

$$
\ell_{1}+\ell_{2}<3.24 \times 10^{31} .
$$

We have just proved the following lemma.

Lemma 4. All solutions to the Diophantine equation (10) satisfy

$$
\ell_{1}+\ell_{2}<3.24 \times 10^{31} \text { and } n<1.22 \times 10^{32} .
$$


4.3. Reducing the bounds. The bounds given in Lemma 4 are to large to carry out meaningful compution. Thus, we need to reduce them. To do so, we apply Lemma 1 as follows.

First, we return to 13 and put

$$
\Gamma_{1}:=\left(\ell_{1}+\ell_{2}\right) \log 10-n \log \alpha-\log \left(\frac{9 a}{d_{1}}\right) .
$$

The inequality 13 can be rewritten as

$$
\left|e^{-\Gamma_{1}}-1\right|<\frac{28}{10^{\ell_{1}}} \text {. }
$$

Assume that $\ell_{1} \geq 2$, then the right-hand side in the above inequality is at most $7 / 25<1 / 2$. The inequality $\left|e^{z}-1\right|<w$ for real values of $z$ and $w$ implies that $z<2 w$. Thus,

$$
\left|\Gamma_{1}\right|<\frac{56}{10^{\ell_{1}}}
$$

which implies that

$$
\left|\left(\ell_{1}+\ell_{2}\right) \log 10-n \log \alpha-\log \left(\frac{9 a}{d_{1}}\right)\right|<\frac{56}{10^{\ell_{1}}} .
$$

Dividing through by $\log \alpha$ gives

$$
\left|\left(\ell_{1}+\ell_{2}\right) \frac{\log 10}{\log \alpha}-n+\left(\frac{\log \left(d_{1} / 9 a\right)}{\log \alpha}\right)\right|<\frac{56}{10^{\ell_{1}} \log \alpha} .
$$

So, we apply Lemma 1 with the data:

$$
\tau:=\frac{\log 10}{\log \alpha}, \quad \mu\left(d_{1}\right):=\frac{\log \left(d_{1} / 9 a\right)}{\log \alpha}, \quad A:=\frac{56}{\log \alpha}, \quad B:=10, \quad 1 \leq d_{1} \leq 9 .
$$

Let $\tau=\left[a_{0} ; a_{1}, a_{2}, \ldots\right]=[3 ; 1,3,1,1,14,1,3,3,6,1,13,3,4,2,1,1,2,3,3,2,2,1,2,5,1,1,39,2, \ldots]$ be the continued fraction expansion of $\tau$. We choose $M:=10^{32}$ which is the upper bound on $\ell_{1}+\ell_{2}$. With the help of Mathematica, we find out that the convergent

$$
\frac{p}{q}=\frac{p_{62}}{q_{62}}=\frac{5067116767207083507605709005080661}{1341009632511071028566373818645201}
$$

is such that $q=q_{62}>6 M$. Furthermore, it yields $\varepsilon>0.0893601$, and therefore either

$$
\ell_{1} \leq \frac{\log ((56 / \log \alpha) q / \varepsilon)}{\log 10}<35,
$$

Thus, we have that $\ell_{1} \leq 35$.

For fixed $0 \leq d_{2}<d_{1} \leq 9$ and $1 \leq \ell_{1} \leq 35$, we return to 16 and put

$$
\Gamma_{2}:=\ell_{2} \log 10-n \log \alpha+\log \left(\frac{d_{1} \cdot 10^{\ell_{1}}-\left(d_{1}-d_{2}\right)}{9 a}\right) .
$$

From the inequality $(16)$, we have that

$$
\left|e^{\Gamma_{2}}-1\right|<\frac{2}{\alpha^{n}}
$$


Since $n>200$, the right-hand side of the above inequality is less than $1 / 2$. Thus, the above inequality implies that

$$
\left|\Lambda_{1}\right|<\frac{4}{\alpha^{n}}
$$

which leads to

$$
\left|\ell_{2} \log 10-n \log \alpha+\log \left(\frac{d_{1} \cdot 10^{\ell_{1}}-\left(d_{1}-d_{2}\right)}{9 a}\right)\right|<\frac{4}{\alpha^{n}} .
$$

Dividing through by $\log \alpha$ gives,

$$
\left|\ell_{2}\left(\frac{\log 10}{\log \alpha}\right)-n+\frac{\log \left(\left(d_{1} \cdot 10^{\ell_{1}}-\left(d_{1}-d_{2}\right)\right) / 9 a\right)}{\log \alpha}\right|<\frac{4}{\alpha^{n} \log \alpha} .
$$

Again, we apply Lemma 1 with the data:

$$
\tau:=\frac{\log 10}{\log \alpha}, \quad \mu\left(d_{1}, d_{2}\right):=\frac{\log \left(\left(d_{1} \cdot 10^{\ell_{1}}-\left(d_{1}-d_{2}\right)\right) / 9 a\right)}{\log \alpha}, \quad A:=\frac{4}{\log \alpha}, \quad B:=\alpha .
$$

We take the same $\tau$ and its convergent $p / q=p_{62} / q_{62}$ as before. We choose $\ell_{2}<10^{32}:=M$. With the help of Mathematica, we get that $\varepsilon>0.0000798749$, and therefore

$$
n \leq \frac{\log ((4 / \log \alpha) q / \varepsilon)}{\log \alpha}<143 .
$$

Thus, we have that $n \leq 143$, contradicting the assumption that $n>200$. Hence, Theorem 1 is proved.

Acknowledgement. The author is supported by the Austrian Science Fund (FWF) projects: F05510-N26 - Part of the special research program (SFB), "Quasi-Monte Carlo Methods: Theory and Applications" and W1230_-Doctoral Program Discrete Mathematics.

\section{REFERENCES}

[1] Alahmadi, A., Altassan, A., Luca, F., and Shoaib, H., Fibonacci numbers which are concatenations of two repdigits, Quaest. Math., To appear, 2019. https://doi.org/10.2989/16073606.2019. 1686439

[2] Bugeaud, Y., Mignotte, M., and Siksek, S., Classical Classical and modular approaches to exponential Diophantine equations I. Fibonacci and Lucas perfect powers, Ann. of Math. (2), 163(2) (2006), 969-1018.

[3] Dujella, A. and Peтhö, A., A generalization of a theorem of Baker and Davenport, Quart. J. Math. Oxford Ser. (2), 49(195) (1998), 291-306.

[4] Gúzman SÁnchez, S. and Luca, F., Linear combinations of factorials and $s$-units in a binary recurrence sequence, Ann. Math. Qué., 38(2) (2014), 169-188.

[5] Matveev, E. M., An explicit lower bound for a homogeneous rational linear form in the logarithms of algebraic numbers II, Izv. Ross. Akad. Nauk Ser. Mat., 64(6):125-180, 2000, in Russian: English translation in Izv. Math., 64(6):1217-1269, 2000.

[6] OEIS Foundation Inc., The On-Line Encyclopedia of Integer Sequences, 2019, https://oeis.org.

[7] Rayaguru, S. G. and Panda, G. K., Balancing numbers which are concatenations of two repdigits, Bol. Soc. Mat. Mex., To appear, 2020. 
Institute of Analysis and Number Theory

Graz University of Technology

Kopernikusgasse 24/II

A-8010 GRAZ

AUSTRIA

E-mail address: mddamulira@tugraz.at; mahadi@aims.edu.gh 